\title{
Smart database concept for power management in an electrical vehicle
}

Mahmoudi Chokri, Flah Aymen, Sbita Lassaad

ENIG National School of Engineering of Gabès, University Of Gabès, Tunisia

\begin{tabular}{l}
\hline \hline Article Info \\
\hline Article history: \\
Received May 24, 2018 \\
Revised Jul 27, 2018 \\
Accepted Aug 16, 2018 \\
\hline
\end{tabular}

Keywords:

Communication

$\mathrm{HEV}$

Management

Power

\begin{abstract}
As world population continues to grow and the limited amount of fossil fuels begin to diminish, it may not be possible to afford the needed amount of energy demanded by the world by only using fossil fuels. Meanwhile, the abundant nature of renewable energy sources brings new beginning for next generations. Greater penetration of electric vehicles will play an important role in building green and healthy world. The main remaining issue to make the switch from conventional to electric vehicle is performance cost; Efficient EVs that can drive for long distances, on single charge, are still expensive for ordinary consumer. To address this range problem, many attempts have been made during last decade. The goal was to conceive a power efficient electric vehicle, capable of managing its energy and reach longer distances. It depends on the electrical architectures and used algorithms. This paper adds new perspective for power Management in EVs; The proposed methodology introduces a new power management architecture based on communication and car learning. The conventional software level in EV has been replaced with self readjustable software. EVs are connected through a database, and can upload or download adjustment parameters while software is running. To take advantage of the new architecture, a new learning technique concept is introduced too, based on Cloud experience exchange between Electric Vehicles. This enhancement aims to build a better EV experience in power management through Cloud sharing and definitely cut with conventional architecture that may have reached its boundaries.
\end{abstract}

Copyright (c) 2019 Institute of Advanced Engineering and Science. All rights reserved.

\section{Corresponding Author:}

Mahmoudi Chokri,

SPEG Research Unit, ENIG National School of Engineering of Gabès, University Of Gabès, Tunisia.

Email: chokri.mahmoudi@gmail.com

\section{INTRODUCTION}

Global warming, climate change, increasing demand on energy, and nations' desire to become green and respectful to next generations have resulted in a need to change electricity production and consumption, including a radical increase in renewable energy sources for daily applications. Information and communication technology (ICT) is already essential in automotive industry. It improves driving safety, performance and comfort. Moreover, these effects go further with electric vehicles: ICT became the backbone of all relevant functions in the vehicle, opening horizons for new electrical architectures.

In last few years, Electric Vehicle started replacing conventional vehicle in many countries, around the world. As entry range EVs are more affordable nowadays, these cars are a smart choice for any end-user, however, autonomy and applications variety remain unsolved [1]. Many attempts to improve Power Management in EV have been done, and many structures have been developed. Today, it is clear that current state of the art is reaching a point where new and significantly different architectures are needed [2]. 
With a better understanding for our needs, and based on similar approaches in other fields such as robotics and computer science, we have a new vision for electric vehicle in the next decade. Here, we formulate the power management control problem and present the control algorithms that can be used to derive the optimal control policy [3]. In our perspective, an Electric Vehicle equipped with learning Power Management Techniques, can enhance its experience over the time by learning new conditions and making new decisions [4]. But if the knowledge is shared between many EVs, it can be a major leap forward. References of various research efforts that have used these algorithms in various configurations are provided in this paper as [5]-[7]. In addition, we are proposing a new approaches that can make innovation in Power Management Architecture and lead other researchers to unexplored ideas

This new architecture is based on a smart networking between EVs running an enhanced version of the learning Power management control algorithm and communicating all the data to a Smart Database. The DB is reinforced with ANNs attribution and selection programs to manage optimal traffic. This new architecture technically offers communication, experience sharing and better power management achievements. We believe it can be a game changer in Automotive Industry.

The remainder of this paper proceeds as follows. After a general introduction, we introduce in Section II the power management control algorithms for Electric Vehicles to date. In Section III we explain the two know methods of Power management Architecture. Then, we detail our proposed contribution in the management of power in an electrical vehicle. Finally, we conclude the exposed work with some future research.

\section{EV AND POWER MANAGEMENT}

Control strategies for hybrid-electric vehicles generally target several simultaneous objectives. The primary one is the minimization of the vehicle fuel consumption, while also attempting to minimize emissions and to maintain or enhance drivability. To date, the power management (PM) system in EVs is basically formed by two layers; High level software-based supervision and low level hardware-based control which can be divided into two control layers low level component and low level control. Both hardware and software control layers works together to optimize PM system in EVs as it is explained in [8], [9].

Major challenge of energy management system (EMS) in an electric vehicle is to assure optimal use and regeneration of the total energy in the vehicle [10]. Regardless of number of sources, the powertrain configureuration, at any time and for any vehicle speed, the control strategy has to determine the power distribution between different energies. When two storage systems or two fuel converters are available additional power distribution between the RESSs and between the fuel converters has to be determined. These decisions are constrained by two factors. First of all, the motive power requested by the driver must always be satisfied up to a maximum power demand already known. Then, charge status must be maintained within, allowing the vehicle to be charge continuously as it is explained in [11]

Many factors can affect the EV performance, such as size, purpose of use, environment, driving style (sporty, soft, moderate or combined). All these factors may lead to a deep and quick discharge rate of the battery and its damage [12].

To keep it healthy and guide it to a slow discharge even when a heavy load is on demand, the electric vehicle is powered by a combination of multiple sources [13]-[15].

The main element is the batteries. Most of the electric vehicles use Lithium ion battery. Lithium ion batteries are environmentally friendly and have higher energy density, longer life span, and higher power density than conventional battery [16]. They have wide application in electric vehicles and other electronics. Since large number of Lithium-ion batteries used in series in electric vehicles so there arises the problems of safety, durability, thermal breakdown and cost, which limits the application of the Lithium ion battery [17]. Some electric vehicles use other kinds of batteries such as Plumb-Acid, Nickel-Cadmium, and lithiumpolymer. The selection of a battery is based on many criteria, such as energy, weight, lifetime, price, voltage, size [18], [19].

To obtain a power boost, super capacitor is used. It has the characteristics of both, capacitor and battery. It can release a large charge in a short period. A super capacitor bank is hence adopted to supply instantaneous charge to assist the main battery in heavy consumption. The super capacitor, under management, can be charged by the main batteries [20], [21].

Recently, many manufacturers accorded more attention to solar panels. They will provide the power management system with an auxiliary electric energy to be used later for battery charging or electronics power supplying as it is explained by [22], [23].

In order to recover kinetic energy lost in vehicle braking electric vehicles can also save energy in stop and go driving through regenerative braking. In this technique, the Electric motor is used as a generator converting the kinetic of the vehicle's motion back to electric energy, rather than dissipating it as heat in the 
brakes. The regenerative braking can recover $50 \%$ to $80 \%$ of the kinetic energy for later use. This is especially valuable for vehicles that stop and start frequently like buses and in-city BEVs [4].

For BEVs and PHEVs, more than source of energy can be used but Grid Power is the main energy source. It allows charging batteries and super capacitors. Many charging modes are available with enhanced charging time. A power management unit based on smart algorithms manages the sources and performs combinations or timing between them to obtain optimal vehicle responsiveness and battery health. This power is transferred to a regular motor control unit, which drives the vehicle.

\section{GENERALITY ON OFFLINE AND ONLINE POWER MANAGEMENT CONTROL} ALGORITHM

Generally, two categories of algorithms are present in the power management for the EV application. The offline power management algorithm, which is based on an optimization Criteria and the online algorithm, which is based on a predictive controller.

The offline algorithm was based on a stochastic optimal control of complex dynamic systems. The problem is formulated as sequential decision making under uncertainty, where a controller is faced with the task of selecting actions in several time steps to efficiently achieve the system's long-term goals. This is was explained by [24]-[26].

Effectively, the system is observed during a completed period of time T known as "the decisionmaking horizon" and can be either finite or infinite. For the finite decision-making horizon problem, the objective is to drive the optimal control policy that minimizes total expected cost criterion, but in major cases, the horizon limit is push to infinite as it is exposed in (1).

$$
J\left(x_{0}\right)=\lim _{T \rightarrow \infty} \frac{1}{T+1} E^{\pi}\left[\sum_{t=0}^{T} k\left(X_{t}, U_{t}\right)\right]
$$

In the offline algorithm, we can also found the Dynamic programming (DP), which is generalized as the main method to analyze sequential decision-making problems, such as deterministic and stochastic optimization and control problems, mini-max problems, and other varied problems [27]. While the nature of these problems may vary widely, their underlying structure is similar to each other and has two principal features: an underlying discrete time dynamic system whose state evolves according to given transition probabilities that depend on the decision taken at each time and a cost function that is additive over time as it is cited in [28], [24].

The second category of the power management algorithms can be called the Online Power Management Control algorithm. The Model predictive control (MPC) is one of the online algorithms it is relies on prediction models to obtain a control action by solving an online optimization problem over a finite horizon. It is often used in constrained regulatory related control problems of large scale multivariable systems, where the objective is to operate the system in a certain desired way [25]. At a specific time " $\mathrm{t}$ ", the actual state of the system is sampled, and control strategy is calculated for a relatively short time horizon $\mathrm{N}$ here, $1(\mathrm{Xt}, \mathrm{Ut})$ is a cost function, as following:

$$
\begin{aligned}
& J=\min _{u_{t} \in U} \sum_{t=k}^{k+N-1} l\left(X_{t}, u_{t}\right) \\
& X_{t+1}=f\left(X_{t}, u_{t}, W_{t}\right), X_{t} \in S: u_{t} \in U
\end{aligned}
$$

Also, Pontryagin's Minimum Principle and ECMS are used. The principle is related to resolving an optimization problem by deriving a set of necessary conditions that must be satisfied by any optimal solution. These conditions become sufficient under certain convexity conditions on the objective and constraint functions [29].

We can found also, the Rule-Based Power Management Control Algorithms. Effectively this method relays on expert experience base to determine fine adjustments to be applied in PMC algorithm. The PMC strategy can be based on fuzzy logic, decentralized adaptive logic, or even new set of rule based PMC algorithms [30], [31].

To optimize EV efficiency, PMC algorithms include a learning mechanism that allows improving performance over time, every single reaction of the driver is considered including driving style, sprint, breaking style, and distances driven. All these collected information build a database specific to the user driving style and there are PM adjustments communicated to driving parameters. This has a major impact on fuel economy and system responsiveness [30]. This principle is included in the Smart / Learning Power Management Control Algorithms.

Int J Pow Elec \& Dri Syst, Vol. 10, No. 1, March 2019: 160 - 169 
GPS enhanced Power Management Control Algorithms. These algorithms are to enhance PMC algorithms using information received from a Global Positioning System (GPS). The algorithm uses data and loads corresponding topography of the road and operates according to preconfigure (ured driving style to minimize fuel consumption. These enhancement algorithms are using driving pattern recognition to automatically select a control algorithm from a bank of six optimized representative driving modes using artificial neural networks (ANNs) [32]. Many works contributed to give a better understanding for Power Management in Electric Vehicle. Depending on powertrain architecture, various PMC algorithms have been introduced [20], [33], [21], [22].

This work focuses in improving learning algorithms and enhancing EV ability to master power management over time and experience. Driving factors and driver behavior has major impact on fuel efficiency, vehicle responsiveness and driving autonomy [29]. Up to now, the research reported few potential works in this category; a multi-modes PMC strategy for parallel HEV proposed in 2002 by Jeon using driving pattern recognition to select a control algorithm automatically from six optimized driving modes using Artificial Neural Networks (ANNs) [34], [35], [14]. Online prediction of future driving cycle based on recorded data was introduced by [36].

The learning strategy was explained by Chen [37] and Salman [38] for parallel HEVs to maximize fuel economy . A learning technique is applied to the cost function in order to adjust parameters in real time. Neuro-dynamic programming was also used in power management control algorithms. Few years later, Kolmanovsky and Dextreit introduced Game Theory Algorithms in power management and experimental results on Land Rover Freelander HEV mark-2 were remarkable [10].

Thus, it is clear that to obtain the best energy consumption, a real-time controller must adapt itself to varying driving circumstances and conditions. In 2011, including driving conditions in power management has been though out [30]. Global Positioning System (GPS) retrieved information were used to determine upcoming topography of the road and adjust PMC parameters. An environment friendly architecture is proposed for HEVs by Li in 2012 to improve efficiency [32]. This concept integrates three modules; a clean energy powertrain, and electrified chassis and intelligent information interaction devices.

\section{PROPOSED CONTRIBUTION IN POWER MANAGEMENT}

The choice of the appropriate topology requires preliminary understanding of vehicle use purposes study of driving cycles, vehicle size and weight, desired performance, and type of application. Once the topology has been set, the second step is the design of an energy management control (EMC) strategy, which is an essential key for an efficient electric vehicle [39]. We can devise the Power management control into tow level.

Low level PM control, which offers a rich range of architectures as: Series HEV is convenient for stop-and-run use, such as city driving. It can recover energy from regenerative breaking and feed batteries. Meanwhile, Parallel HEV has a weak battery capacity [38]. The ICE and EM complement each other while driving. Thus, it can be reliable in either city or highway. This kind of structure gets a better efficiency because of the reduced battery pack and small electric motor. The main area, both previous architectures cannot cover is the precise control strategy. Thus tow complex configuration are used; Series-parallel HEV and Complex HEV. PHEV sustains longer in EM mode than ICE mode. It is suitable for both city and highway, and shares the same advantages and disadvantages of a regular HEV [29][21][40]. For BEVs, inwheel drive configure (uration is most suitable for city use due to lightweight and frequent stop-and-run situations. BEVs are designed mainly for short distance autonomy despite of minimal energy loss in transmission. Handling of BEVs will be affected by the new wheel configuration and increase of its weight.

In high level, the power management controller would take advantage of different algorithms developed for this purpose, but also takes even more advantages from enhancing algorithms, weather conditions, weather forecast, GPS position and driving experience [31].

\subsection{Intelligent power management architecture}

The idea is simple; learning PMC algorithm can be improved; EVs would be able to learn from each other through communication; a user experience exchange database, encrypted to respect drivers' privacy [31] [41]. By providing more accurate and up to date data to power management system, fuel economy can be improved, reducing pollutant emissions, as well as extending battery lifetime and range.

In EV Power Management, main introduced algorithms, whether they are Offline or Online, are generally applied locally to optimize energy efficiency. No potential studies were introduced in this category except safety through Vehicle to Vehicle communication [42]. The new architecture introduced in this work aims to explore vehicles' communication to improve power management, the general principle of this new proposition can be summarized in Figure 1. It is based on two levels. 


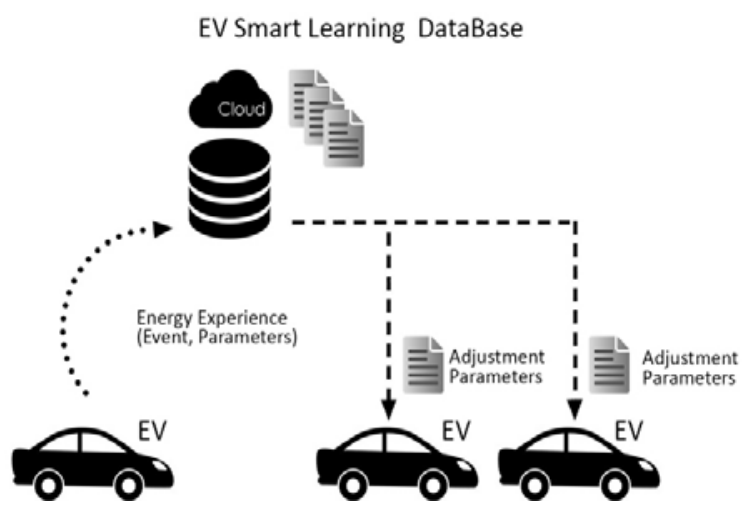

Figure 1. Smart learning architecture concept

The first level; where vehicle optimizes power management using self-learning techniques and builds its own Energy Experience (E.EX). This unique E.EX will take advantage of various driving situations. The way the vehicle adjusts PMC algorithm parameters is related to its driving experience; the vehicle learns from driver's behavior and mood, GPS position, road conditions, time conditions, weather conditions. All these information will help to build a rich energy experience and to set fine selection criteria for the next level. Now according to each driving situation, a new set of adjustment parameters is provided. Through time, Vehicle will develop a unique understanding for energy management. Its experience will grow, while adaptation and responsiveness will get better.

The second level in this new architecture will take advantage of single vehicle achievements in energy experience to build a Smart Learning Database (SLDB). In this architecture, a cloud based Database will collect EV Energy Experiences from different connected vehicles to be sort by events.

Energy experiences are uploaded to the base and sort by very fine event criterion. Through a comprehensive power management data base solution, a vehicle detecting similar conditions during its path sends an assist request to the DB. This request will be identified and feedback will be downloaded to vehicle if available. Therefore, it gets advantage of valuable previous energy experience of another vehicle. By downloading adjustment algorithm parameters for power management, it obtains optimal, know to date, energy economy and vehicle responsiveness immediately.

\subsection{Description of a sequence}

In this architecture, we examine all information collected by vehicle. This contributes in building vehicle decision. These information constitute an event; e.g. An electric vehicle being driven by a female driver, having a happy mood, from a GPS position A to B, in specific road conditions, in summer, in specific weather condition and temperature, the ride was in a specific date \& Time a weekend, a feast day, in the morning, traffic conditions during that day a traffic jam occurred. The vehicle status indicates fully charged batteries, with half fuel tank left. According to this event in space and time, with this gender of driver, in this mood, working with all mentioned conditions, we generate a unique set of adjustment parameters. In vehicle recorded data this event may occurs probably in the future. Drivers generally select unconsciously same itinerary to link between two points A and B. some drivers may know shortcuts during peak time in order to avoid jam and improve fuel economy. All these information can bring assistance to new vehicles in this area to define, in a comprehensive way, the decision to make, itinerary to suggest and parameters to set in its PMC algorithm.

\subsection{Novel energy experience concept}

Adaptive and Smart Learning algorithms estimate their optimal values in real-time in order to satisfy the charge-sustaining constraint and to achieve best performance [25]. Intelligent decision taken seems to be only related to vehicle. However, different circumstances may lead to complete different decisions and various results.

In a second step, Energy Experience layers are introduced; Information such as engine status, batteries' rate, etc. are provided as conventional from the Electric vehicle. To build a rich experience, information from driver and environment such as driver gender, mood, GPS location, weather and road conditions are added to create an Event.

Int J Pow Elec \& Dri Syst, Vol. 10, No. 1, March 2019: 160 - 169 
The Smart learning Power Management Control Algorithm runs separately to predict new adjustment parameters which are suitable for this event. The combination between the event and the parameters constitutes the energy experience.

To get Database assistance, a request mentioning the current event is formulated and sent. The vehicle should have an inexistent or poor experience related to the occurred situation. Basically, two scenarios are faced. In first case, according to the sent request, no similar situations are found. The Data-Base cannot afford adjustment parameters for the Electric Vehicle. Thus, no assistance is offered. As presented in Figure 2.

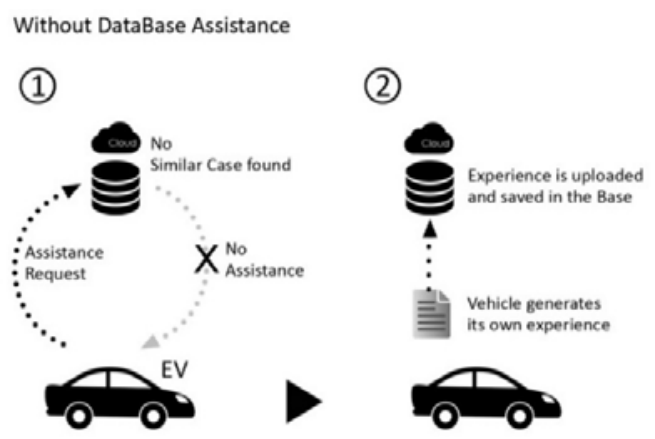

Figure 2. PM without smart data-base assistance

The vehicle generates its own adaptive parameters for the faced situation aiming to optimize power management. These Parameters, coupled with the event description are uploaded as an experience for future use. The Data-Base provides Electric Vehicle with adjustment parameters. The vehicle utilizes optimized parameters for the faced situation. These Parameters will allow to obtain immediate optimal PM, better vehicle responsiveness and to improve immediately fuel economy for a longer ride range. This phenomenon is exposed in Figure 3.

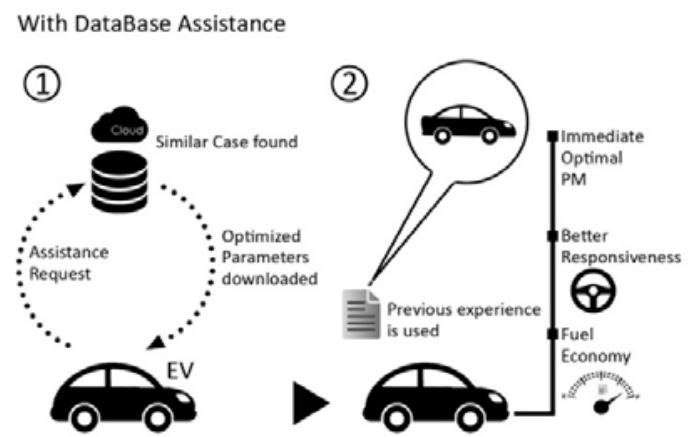

Figure 3. PM with smart database assistance

As well as Electric Vehicles intend to improve knowledge through local and cloud experience sharing, the Data-Base operates identically. Each time the uploaded parameters define a better efficiency for an existing experience compared to the saved data, the new parameters replace the old ones.

Various information can be delivered by vehicle sensors to create the event. In order to simplify systems and reduce complexity, we use structure sharing strategy. Which involves that same physical structure or sensor can be shared by different functions and provide needed information. E.g. same camera can be used in Drowsiness Detection System (DDS) to alert driver sleepiness and as a face analyzer to determine driver mood. Same sensors used in Lane Keeping Assist System (LKAS) [41] to oblige driver to keep hands on steering wheel, can provide heartbeats rate and determine if the driver is nervous. Previous researches as [39], [43] have highlighted the prominence of structure sharing in Electric Vehicle. 
Therefore, for complex system design, few studies were introduced. In 2012, Keqiang Li proposed a comprehensive design for structure sharing; In order to minimize sensors onboard and simplify architecture, same physical equipment can provide information for different functions or features in a vehicle. This improves efficiency for complex architecture at the lowest cost. In our case, we are more interested in multisensory principle, which aims to reduce the number of onboard sensors. As shown in Figure 4, sensors are shared among ordinary functions such as comfort or security and Power Management Learning process. E.g., GPS antenna provides location information to onboard navigation system for turn-by-turn assistance and, simultaneously, calculates coordinates which are useful to build the energy experience or to retrieve assistance from the Smart Database. Information come from vehicle as well as driver and environment. The multisensory structure sharing should be controlled. Depending on valuation, to reduce cost, further redundancy or reliability, the structure will be defined and designed [44].

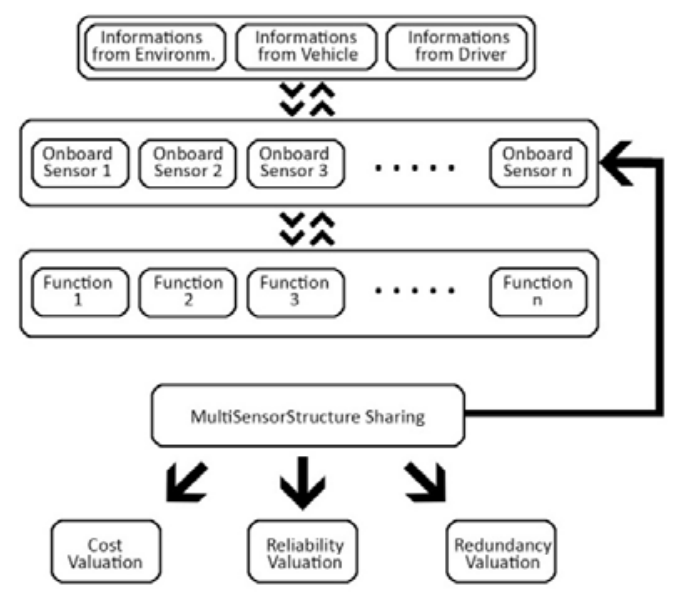

Figure 4. Multi-sensor structure sharing

\subsection{Novel Algorithm Structure and Layout}

A cloud database typically runs on a cloud-computing platform, such as Salesforce, Go Grid, and Microsoft Azure. As deployment, the choice of Cloud databases is the independency due to different manufacturers' competition and professional secret anonymity. By using secured and independent virtual machines, decision about data model will be taken in further work whether it should be SQL-based No-SQL database model. As a general layout, Energy experiences are uploaded systematically to the base. When information is requested in normal or predictive approach, database seeks the convenient experience base on similarity in events. Research and experience selection will be optimized with Artificial Neuronal Networks (ANNs). These Software functions aim to take advantages of Cloud Computing revolution.

In high supervisory Power Management Layer (PML), many algorithms have been developed. Depending on powertrain architecture, mainly five techniques delivered intended results and proved reliability. In this section, we will go through a brief mention of recent efforts introduced in both offline and online algorithms. And will introduce an enhanced algorithm structure to go with the novel architecture, as presented in Figure 5.

When we aimed to rebuild the power management experience, we set an enhanced online PMC algorithm that inherit smart learning feature from existing Learning PMC algorithm and localization feature from "GPS Enhanced Algorithm". The Novel Algorithm takes advantage of the learning technique, but sets the optimal parameters in a vector collecting all the conditions in which the optimal parameters are found. This parameter's vector is called Optimal Experience Vector. Instead of enhancing just one vehicle, the vector integrates the EV information such as brand, model, powertrain, manufacturer to be a referenced Experience Vector, then sent to our Smart cloud database for saving.

As we use different EVs, different Energy experiences are communicated to the Database building and important decision center ready for use. The running algorithm of the proposed method is presented in Figure 7. The initializing structure of the vehicle is also exposed in Figure 6. 


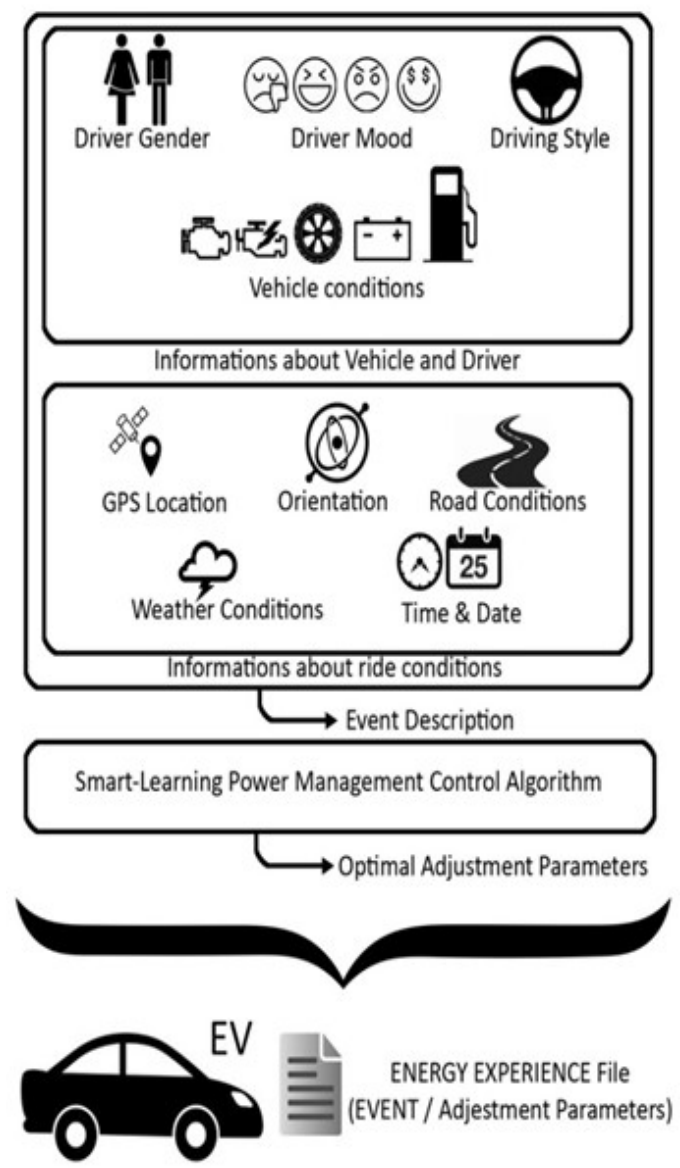

Figure 5. Smart learning architecture concept

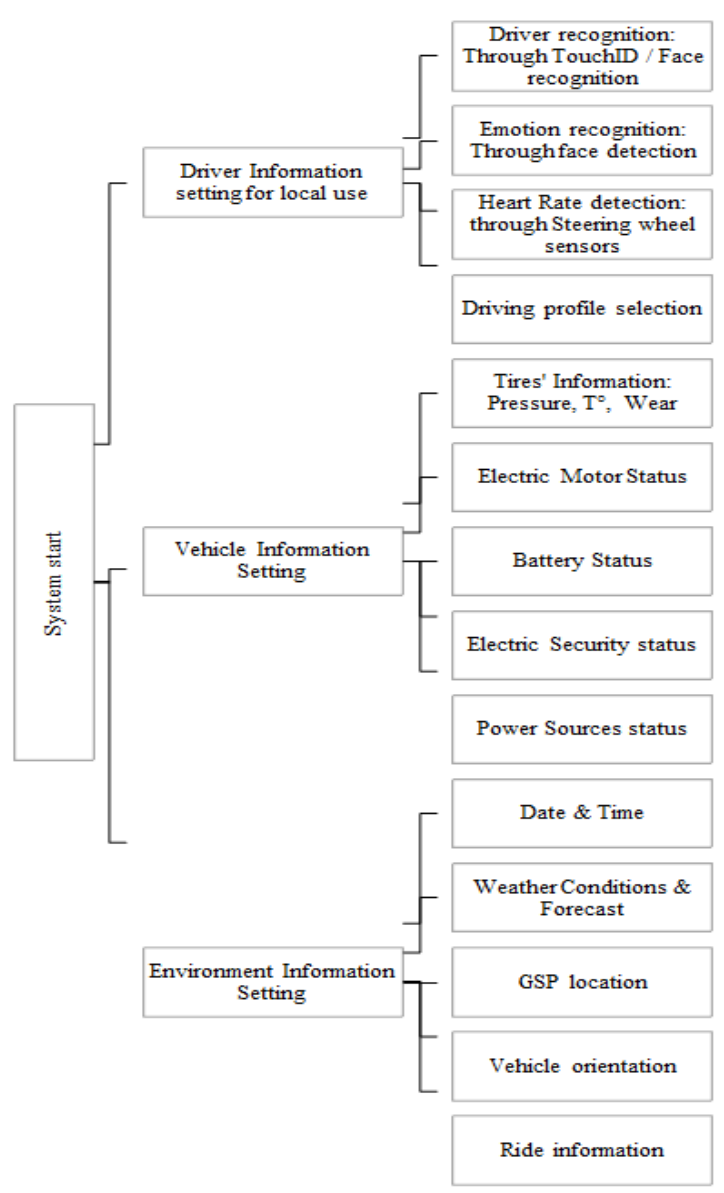

Figure 6. Vehicle initializing structure

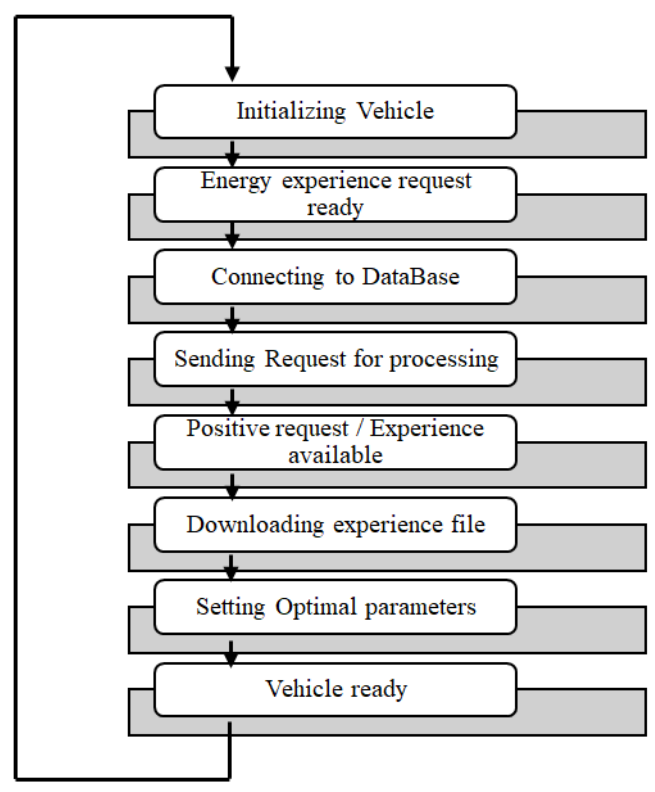

Figure 7. Flowchart or a start sequence 


\section{CONCLUSION}

Connecting vehicles represents an important trend for the next generation. The key technology in the Smart Learning Architecture is experience sharing between vehicles to improve Self learning and enhance power management. Providing green solutions with environmental friendly goals should not in any way compromise in vehicle responsiveness or driving style. The present paper describes a new method of power managing in Electric Vehicles. The work carried out determine the possibility of providing a new architecture that can afford power management, networking and connectivity between EVs. As a next step in this work, simulations results will be published in order to reinforce our Architecture.

\section{REFERENCES}

[1] E. Helmers and P. Marx, "Electric cars: technical characteristics and environmental impacts," Helmers and Marx Environmental Sciences Europe, vol. 24, pp. 1-15 2012.

[2] M. A. Hannan, F. A. Azidin, and A. Mohamed, "Hybrid electric vehicles and their challenges: A review," Renewable and Sustainable Energy Reviews, vol. 29, pp. 135-150, Jan. 2014.

[3] J. Z. Pei, Y. X. Su, and D. H. Zhang, "Fuzzy energy management strategy for parallel HEV based on pigeoninspired optimization algorithm," Sci. China Technol. Sci, vol. 60, pp 425-433, 2017.

[4] C. Mahmoudi, A. Flah, and P. Lassaad SBITA, "Novel concept of Power Management Architecture based on Smart EV Learning DataBase," Recent Adv. Electroscience Comput. J., pp. 191-196, 2016.

[5] Z. Wei, J. Xu and D. Halim, "HEV Energy Management Fuzzy Logic Control Based on Dynamic Programming," 2015 IEEE Vehicle Power and Propulsion Conference (VPPC), Montreal, QC, pp. 1-5, 2015.

[6] C. K. Samanta, S. K. Padhy, S. P. Panigrahi and B. K. Panigrahi, "Hybrid swarm intelligence methods for energy management in hybrid electric vehicles," in IET Electrical Systems in Transportation, vol. 3, pp. 22-29, March 2013.

[7] M. Irfan, Machmud Effendy, Nur Alif, Lailis S, Ilham Pakaya, Amrul Faruq, "Performance Comparison of Fuzzy Logic and Proportional-integral for an Electronic Load Controller," International Journal of Power Electronics and Drive System (IJPEDS), vol. 8, pp. 1886-1893, December 2017.

[8] F. R. Salmasi, "Control Strategies for Hybrid Electric Vehicles: Evolution, Classification, Comparison, and Future Trends," in IEEE Transactions on Vehicular Technology, vol. 56, pp. 2393-2404, Sept. 2007.

[9] L. Xu, J. Hua, X. Li, Q. Meng, J. Li and M. Ouyang, "Control strategy optimization of a hybrid fuel cell vehicle with braking energy regeneration," 2008 IEEE Vehicle Power and Propulsion Conference, Harbin, pp. 1-6, 2008.

[10] C. Dextreit and I. Kolmanovsky, "Approaches to energy management of hybrid electric vehicles: Experimental comparison," inProc. UKACC Int. Conf. Control, pp. 1-6, 2010.

[11] P. Pisu and G. Rizzoni, "A Comparative Study Of Supervisory Control Strategies for Hybrid Electric Vehicles," in IEEE Transactions on Control Systems Technology, vol. 15, pp. 506-518, May 2007.

[12] Siti Khalidah Rahimi, Zarafi Ahmad, Erwan Sulaiman, Enwelum Mbadiwe I, Syed Muhammad Naufal Syed Othman, "Performance Analysis of 12Slot with Various Rotor Pole Numbers HE-FSM for HEV Application," International Journal of Power Electronics and Drive System (IJPEDS), vol. 8, pp. 1886-1893, December 2017.

[13] E. Biondi, C. Boldrini and R. Bruno, "Optimal charging of electric vehicle fleets for a car sharing system with power sharing," 2016 IEEE International Energy Conference (ENERGYCON), Leuven, pp. 1-6, 2016.

[14] P. Pisu, K. Koprubasi and G. Rizzoni, "Energy Management and Drivability Control Problems for Hybrid Electric Vehicles," Proceedings of the 44th IEEE Conference on Decision and Control, Seville, Spain, pp. 1824-1830, 2005.

[15] C. Mahmoudi, A. Flah and L. Sbita, "Prototype design of a compact plug-in solar electric vehicle application for smart power management architecture," 2017 International Conference on Green Energy Conversion Systems (GECS), Hammamet, pp. 1-4, 2017.

[16] N. H. F. Ismail and S. F. Toha, "State of charge estimation of a Lithium-ion battery for electric vehicle based on particle swarm optimization," 2013 IEEE International Conference on Smart Instrumentation, Measurement and Applications (ICSIMA), Kuala Lumpur, pp. 1-4, 2013.

[17] D. Notter et al., "Contribution of Li-Ion Batteries to the Environmental Impact of Electric Vehicles," Environ. Sci. Technol., vol. 44, pp. 6550-6556, 2010.

[18] Bi. Emil, M. M. Juan, and M. Henrik, "Optimal charging of an electric vehicle using a Markov decision process," Applied Energy vol. 123, pp. 1-12, 2014.

[19] C. Tsai and C. Ting, "Evaluation of a multi-power system for an electric vehicle," ICCAS 2010, Gyeonggi-do, pp. 1308-1311, 2010.

[20] M. Salazar and N. Ertugrul, "Potential enhancements for vehicle electrical power management systems in military vehicles," 2013 Australasian Universities Power Engineering Conference (AUPEC), Hobart, TAS, pp. 1-6, 2013.

[21] M. Choi, J. Lee and S. Seo, "Real-Time Optimization for Power Management Systems of a Battery/Supercapacitor Hybrid Energy Storage System in Electric Vehicles," in IEEE Transactions on Vehicular Technology, vol. 63, no. 8, pp. 3600-3611, Oct. 2014.

[22] L. Rosario, P. C. K. Luk, J. T. Economou and B. A. White, "A Modular Power and Energy Management Structure for Dual-Energy Source Electric Vehicles," 2006 IEEE Vehicle Power and Propulsion Conference, Windsor, pp. 1-6, 2006.

Int J Pow Elec \& Dri Syst, Vol. 10, No. 1, March 2019: 160 - 169 
[23] F. R. Salmasi, "Control Strategies for Hybrid Electric Vehicles: Evolution, Classification, Comparison, and Future Trends," in IEEE Transactions on Vehicular Technology, vol. 56, no. 5, pp. 2393-2404, Sept. 2007.

[24] A. A. Malikopoulos, "Supervisory Power Management Control Algorithms for Hybrid Electric Vehicles: A Survey," in IEEE Transactions on Intelligent Transportation Systems, vol. 15, no. 5, pp. 1869-1885, Oct. 2014.

[25] N. Chen, T. Q. S. Quek and C. W. Tan, "Optimal charging of electric vehicles in smart grid: Characterization and valley-filling algorithms," 2012 IEEE Third International Conference on Smart Grid Communications (SmartGridComm), Tainan, pp. 13-18, 2012.

[26] K. Clement, E. Haesen and J. Driesen, "Coordinated charging of multiple plug-in hybrid electric vehicles in residential distribution grids," 2009 IEEE/PES Power Systems Conference and Exposition, Seattle, WA, pp. 1-7, 2009.

[27] I. Koohi and V. Z. Groza, "Optimizing Particle Swarm Optimization algorithm," 2014 IEEE 27th Canadian Conference on Electrical and Computer Engineering (CCECE), Toronto, ON, pp. 1-5, 2014.

[28] Y. He, B. Venkatesh and L. Guan, "Optimal Scheduling for Charging and Discharging of Electric Vehicles," in IEEE Transactions on Smart Grid, vol. 3, pp. 1095-1105, Sept. 2012.

[29] X. Li and S. S. Williamson, "Assessment of Efficiency Improvement Techniques for Future Power Electronics Intensive Hybrid Electric Vehicle Drive Trains," 2007 IEEE Canada Electrical Power Conference, Montreal, Que., pp. 268-273, 2007.

[30] A. A. Malikopoulos, "Real-Time, Self-Learning Identification and Stochastic Optimal Control of Advanced Powertrain Systems," in Ann Arbor, MI, USA, 2011.

[31] M. Saleem, G. A. Di Caro, and M. Farooq, "Swarm intelligence-based routing protocol for wireless sensor networks: Survey and future directions," Inf. Sci. (Ny)., vol. 181, pp. 4597-4624, 2011.

[32] Li Guoyong and Yan Fang, "NN-based fuel injection control system for hybrid fuel engine," 2012 IEEE Symposium on Electrical \& Electronics Engineering (EEESYM), Kuala Lumpur, pp. 336-340, 2012.

[33] M. Tsai, C. Tseng and Y. Lin, "Power management and control of an electric vehicle with auxiliary fuel cell and wind energies," 2013 IEEE International Conference of IEEE Region 10 (TENCON 2013), Xi'an, pp. 1-4, 2013.

[34] YLavanya Dhanesn, P. Murugesan, "A Novel Approach in Scheduling Of the Real- Time Tasks In Heterogeneous Multicore Processor with Fuzzy Logic Technique For Micro-grid Power Management," International Journal of Power Electronics and Drive System (IJPEDS), vol. 9, pp. 80-88, March 2018.

[35] J. Lai and D. J. Nelson, "Energy Management Power Converters in Hybrid Electric and Fuel Cell Vehicles," in Proceedings of the IEEE, vol. 95, no. 4, pp. 766-777, April 2007.

[36] G. Paganelli, M. Tateno, A. Brahma, G. Rizzoni and Y. Guezennec, "Control development for a hybrid-electric sport-utility vehicle: strategy, implementation and field test results," Proceedings of the 2001 American Control Conference. (Cat. No.01CH37148), Arlington, VA, USA, pp. 5064-5069 vol.6, 2001.

[37] C. K. Chan, K. W. E. Cheng and S. L. Ho, "Development of Packaging and Electrical Interfacing for Electrical Vehicles," 2006 2nd International Conference on Power Electronics Systems and Applications, Hong Kong, pp. 234-240, 2006.

[38] M. Salman, N. J. Schouten, and N. A. Kheir, "Control strategies for parallel hybrid vehicles," Proceedings of the 2000 American Control Conference. ACC (IEEE Cat. No.00CH36334), vol. 1, pp. 524-528, 2000.

[39] S. Hajforoosh, M. A. S. Masoum, and S. M. Islam, "Real-time charging coordination of plug-in electric vehicles based on hybrid fuzzy discrete particle swarm optimization," Electr. Power Syst. Res., vol. 128, pp. 19-29, November 2015

[40] P. Elbert, T. Nüesch, A. Ritter, N. Murgovski, and L. Guzzella, "Engine On/Off Control for the Energy Management of a Serial Hybrid Electric Bus via Convex Optimization," IEEE Transactions on Vehicular Technology, vol. 63, pp. 3549-3559, 2014.

[41] Y. Cao et al., "An Optimized EV Charging Model Considering TOU Price and SOC Curve,"IEEE Transactions on Smart Grid, vol. 3, pp. 388-393, 2012.

[42] A. Fotouhi, D. J. Auger, K. Propp, S. Longo, and M. Wild, "A review on electric vehicle battery modelling: From Lithium-ion toward Lithium-Sulphur, " Renewable and Sustainable Energy Reviews, vol. 56, pp. 1008-1021, 2016.

[43] A. Panday and H. O. Bansal, "A Review of Optimal Energy Management Strategies for Hybrid Electric Vehicle," Int. J. Veh. Technol., vol. 2014, pp. 1-9, 2014.

[44] Z. Chen, C. C. Mi, R. Xiong, J. Xu, and C. You, "Energy management of a power-split plug-in hybrid electric vehicle based on genetic algorithm and quadratic programming," Journal of Power Sources, vol. 248, pp. 416-426, 2014. 